\title{
Generation of Cardiomyocytes in Pipe- Based Microbioreactor Under Segmented Flow
}

\author{
Dimitry Spitkovsky ${ }^{a}$ Karen Lemke ${ }^{b}$ Tobias Förster ${ }^{b}$ Robert Römer ${ }^{b}$ \\ Stefan Wiedemeier ${ }^{b}$ Jürgen Hescheler ${ }^{a}$ Agapios Sachinidis ${ }^{a}$ Gunter Gastrock ${ }^{b}$
}

aCenter of Physiology and Pathophysiology, Institute of Neurophysiology, University of Cologne,

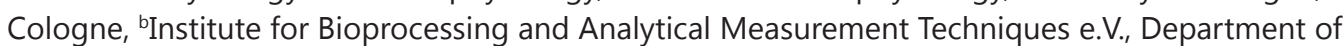
Bioprocess Engineering, Heilbad Heiligenstadt, Germany

\section{Key Words}

ES cells • Cardiomyocytes $•$ Differentiation $\bullet$ Segmented flow $•$ Microbioreactor

\begin{abstract}
Background/Aims: Embryonic stem (ES) cells have got a broad range differentiation potential. The differentiation is initiated via aggregation of non-differentiated ES cells into embryoid body (EB) capable of multi-lineage development. However experimental variables present in standard differentiation techniques lead to high EB heterogeneity, affecting development into the cells of desired lineage, and do not support the process automatization and scalability. Methods: Here we present a novel pipe based microbioreactor (PBM) setup based on segmented flow, designed for spatial maintenance of temperature, nutrition supply, gas supply and sterility. Results: We verified PBM feasibility for continuous process generating cardiac cells starting from single ES cell suspension followed by EB formation for up to 10 days. The ES cells used in the study were genetically modified for cardiac-specific EGFP expression allowing optical monitoring of cardiomyocytes while EBs remained within PBM for up to 10 days. Efficiency of cardiac cells formation within PBM was similar compared to a standard hanging drop based protocol. Conclusion: Our findings ensure further development of microfluidic bioreactor technology to enable robust cardiomyocytes production for needs of drug screening, tissue engineering and other applications. authors. A. Sachinidis and G. Gastrock contributed equally to this manuscript and should be considered as co - last authors. 


\section{Introduction}

Further advances in cell technologies are important for the development of adequate in vitro cell systems supporting drug screening and toxicology assays replacing animal tests, as well as for the progress in regenerative medicine. Novel tools providing a strict environmental control are required to address issues of high complexity, variability and scalability of the potential assays. It is well established that minor alterations in the cell adhesion, medium compositions and physicochemical conditions may have a dramatic impact on the cell properties. Miniaturization of the cell cultivation is supporting a better microenvironmental control, robustness, efficiency and reproducibility in diverse applications. Assay miniaturization is significantly reducing assay costs associated with high throughput pharmacological and toxicological screenings. It could contribute to manufacturing of building blocks for tissue engineering. Miniaturized systems are available in both twodimensional (2D) and three-dimensional (3D) applications [1]. Mammalian cell cultivation in $3 \mathrm{D}$ is preferable as it supports cell maintenance under more physiologically relevant conditions better reflecting in vivo situation. The 3D cell growth can be achieved either via the cells maintenance on adherent substrates and extracellular matrixes or via surface free cultivation.

Scaffold-free approach can be illustrated by EB formation from ES cells. There are two general modifications of such approach based on either batch suspension culture or cell suspension hanging drop protocol [2], the later method is more reproducible due to reduction of the EB heterogeneity. However an original hanging drop protocol has got some limitations in scalability and the droplet stability. High throughput compatible alternative techniques capable to support scaffold-free 3D cell building blocks has been developed recently addressing shortcoming of original hanging drop protocol. It was achieved after developing a hanging drop plate approach allowed simplified liquid handling procedures and compatibility with high-throughput screening instruments $[3,4]$. Other developments are based on surface micro patterning allowing 3D compartments. A silicon wafer-based microfabrication technology was used to generate surfaces that permit the production of hundreds to thousands of hEB per $\mathrm{cm}^{2}$ of the working surface areas and this approach supported synchronization of spatial and temporal EB development [5]. It permits batch process of EBs, but lacks ability to generate diverse environments within individual compartments. Microfabrication based on polyethylene glycol offered control of such parameters as size, shape, and homogeneity [6]. EBs formation was undertaken in relatively easy handled stencils providing good spheroids shape and easy scaling opportunity [7]. Microfluidics device was applied to control the EB formation in a format compatible for ES cell processing on a chip, although initially the ES cells formed cylindrical aggregates within thin channels [8]. Another 3D microfluidic platform used co-flow arrangement with an interesting potential for manipulation within different areas of the same EB [9]. Single cell cultivation can be performed applying e.g. the segmented-flow technique. Segmented-flow based bioreactors offer additional advantages of miniaturized closed systems with better environmental control and higher degree of uniformity. They are based on the generation and manipulation of liquid sample droplets embedded in a hydrophobic transport medium within capillary systems. So far segmented-flow bioreactors have been realized for several biological models. Discrete microbial populations within $60 \mathrm{~nL}$ droplets could rapidly grow reaching density observed in conventional flask cultures $[10,11]$. Such system was applied for the bacteria toxicity screening with selected substances [12]. Segmented flow bioreactor was applied to multi-cellular system. Zebrafish embryos were introduced into tubes with $1.2 \mathrm{~mm}$ inner diameter, and the embryos development was observed over period of 80 hours [13].

Recently segmented flow bioreactor was introduced for droplet generation for selfassembly or scaffold-based 3D cell cultivation [14].

We have demonstrated utility of a novel PBM platform based on the segmented flow principle for generation and expansion of cardiomyocytes from EBs. At the initiation phase

\section{KARGER}


Spitkovsky et al.: Generation of Cardiomyocytes in Microbioreactor

the PBM were loaded with embryonic stem cell suspension. The PBM supported formation and further expansion and differentiation of viable EBs. The EBs could be continuously cultivated within PBM for up to 10 days providing supportive environment for EB steady growth and cardiomyocyte differentiation.

\section{Materials and Methods}

Pipe based bioreactor using segmented flow principles

The principle of segmented flow is based on the immiscibility of at least two fluids, e.g. water and oil. Consequently, in microchannels with hydrophobic surfaces, water forms droplets separated by the oil phase. Each droplet can be considered as microbioreactor if fulfilling several requirements. First of all these microbioreactors should be maintained under aseptic conditions, and therefore the pipe system should withstand adequate sterilization procedures. Additionally, the pipe system should permit a continuous gas supply of the microbioreactors. Importantly, the separation fluid has to be not toxic to the cells and should not interact with the cell culture medium. Moreover, a useful setup should allow medium exchange, medium sampling, addition of medium or drugs and different readout options. Satisfying all these requirements using highly integrated microsystems would be a challenging task and cause high expenses. An alternative approach would be replacing such integrated microsystem by single functional modules which can be realized by simple microfabrication processes or by precision engineering. These modules could be connected by tubes, representing the "microchannels". The tubes must possess a hydrophobic surface, which is guaranteed in the case of e.g. Polytetrafluoroethylene (PTFE) tubes. Besides hydrophobicity PTFE tubes are gas permeable and sufficiently transparent for optical readout. These properties make PTFE tubes applicable for droplet based cell cultivation as well as for optical detection of the cells inside the droplets. Thus, PTFE tube itself could be considered as a functional module. Figure 1A shows such coil shaped module. Besides the gas permeability of the PTFE tubes the separation fluid must be able to store and transport gas (usually air and $\mathrm{CO}_{2}$ ). It could be assured by using Perfluorodecalin (PFD) as a separation fluid. PFD possesses features of a low toxicity and a high oxygen storage capacity as $49 \mathrm{~mL}$ of oxygen per 100

Fig. 1. PBM overview and basic set up. (A) Principle of gas exchange between PBM and an environment; (B) Basic setup placed under a clean bench; (C) Two capillary probe for $800 \mathrm{~nL}$ droplet generation within $1 \mathrm{~mm}$ coil (left hand side) and the $1 \mathrm{~mm}$ coil for cultivation of cells (right hand side). The enlarged section of the right hand side picture shows the droplets inside the PTFE tube.
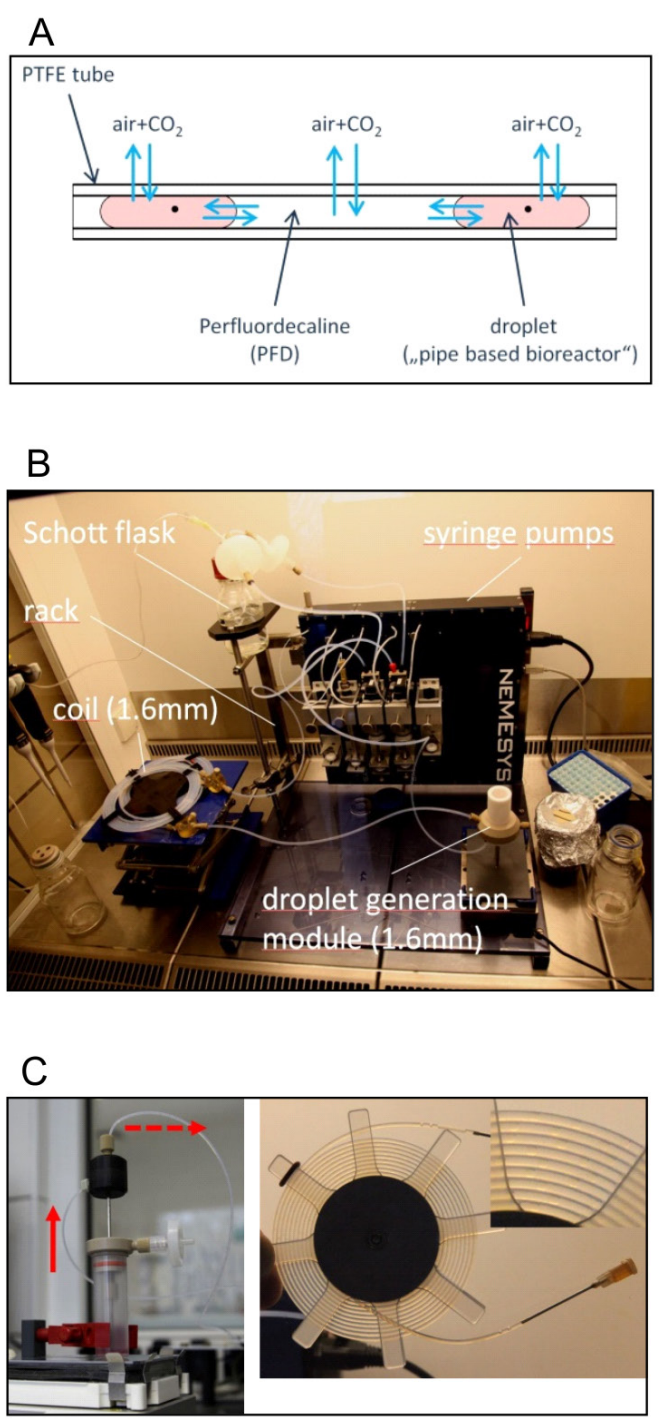


\section{Cellular Physiology Cell Physiol Biochem 2016;38:1883-1896

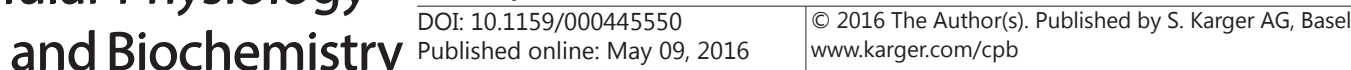

Spitkovsky et al.: Generation of Cardiomyocytes in Microbioreactor

$\mathrm{mL}$ of PFD at $25^{\circ} \mathrm{C}$. Due to high oxygen storage capacity PFD was used as a component of transport medium for organ preservation [15].

The modularity of the system allowed combining the functional modules for different tasks. For example, the setup for droplet generation was built up of two syringe pumps, the droplet generation module, a shaker, and the coil for cell cultivation. The droplet volume could be chosen from $60 \mathrm{~nL}$ up to 20 $\mu \mathrm{L}$ by varying the syringe pumps flow rates and by using different droplet generation modules and different PTFE tubes, respectively.

\section{Basic setup}

The basic setup was built up as a rack at which 6 independently working syringe pumps (cetoni GmbH, http://www.cetoni.de) were mounted. Depending on the procedures the different functional modules (described later) were mounted at the rack and connected to the syringes ( $5 \mathrm{~mL}$ and $25 \mathrm{~mL}$ glass syringes, ILS GmbH, http://www.microsyringes.com/) with PTFE tubes (1.0 mm inner diameter, Jasco GmbH, http:// www.jasco.de/, and $1.6 \mathrm{~mm}$ inner diameter, YMC GmbH, http://www.ymc-europe.com/). Additionally, a $250 \mathrm{~mL}$ Schott flask was mounted at the rack served as reservoir for PFD. PFD was used as the a separation medium splitting the droplets in the tubes into individual compartments. PFD was continuously supplied with a gas mixture of $95 \%$ air and $5 \% \mathrm{CO}_{2}$. The Schott flask was connected via two $1.6 \mathrm{~mm}$ PTFE tubes and two hydrophobic sterile filters (Sartorius GmbH, http://www.sartorius.de/) to two $25 \mathrm{~mL}$ syringes. The positions of the droplets during the operator controlled transfer of $800 \mathrm{~nL}$ into $20 \mu \mathrm{L}$ droplets were detected optically using a digital camera (DigiMicro Profi, dnt Drahtlose Nachrichtentechnik GmbH, http://www.dnt. de). All modules and elements of the basic setup interacting with cells and cell medium were sterilized by autoclaving. During the experiment the rack was placed under the clean bench (Fig. 1B).

Both, the control software for the syringe pumps and the camera control software were installed on a PC with the operation system "Windows 7". The PC, a computer display, a keyboard and a mouse were situated outside the clean bench.

\section{Generation of $800 \mathrm{~nL}$ droplets containing cell suspension}

The generation of $800 \mathrm{~nL}$ droplets was performed using a two capillary probe based on the principle of a "double lumen catheter" [16, 17]. The probe was adapted to a $10 \mathrm{~mL}$ Casy 1 cup (http://www.roche. com). The cup was filled with $2 \mathrm{~mL}$ of the ES cell suspension, and the probe was mounted in such way that it protruded into the cell suspension. ES cells were used at the concentration of 625,000 cells $/ \mathrm{mL}$ allowing 500 cells into $800 \mathrm{~nL}$ droplet. A hydrophobic filter was used avoiding pressure variations during the droplet generation. The cup was mounted on a plate which was positioned on a shaker (BioShake IQ, Qinstruments $\mathrm{GmbH}, \mathrm{http}: / /$ www.bioshake.com/). The cup was shaken at the oscillation frequency of $300 \mathrm{rpm}$ during the droplet generation keeping the cells in the homogeneous suspension. Droplet generation was performed by pumping PFD into the cup and contemporary sucking droplets separated by the PFD out of the cup (see red arrow for separation fluid and broken red arrow for segmented microbioreactors in Fig. 1C, left hand side). The droplets were guided into $1.0 \mathrm{~mm}$ inner diameter PTFE tube (Fig. 1C, right hand side), creating a coil of $2 \mathrm{~m}$. The coil was sealed at both ends with canulas and incubated for required time in a humidified atmosphere of $5 \% \mathrm{CO}_{2}$ at $37^{\circ} \mathrm{C}$. The coils of different length could be adopted if required.

\section{Generation of $20 \mu \mathrm{L}$ droplets with cell cultivation medium}

The module for $20 \mu \mathrm{L}$ droplet generation is shown in Fig. 2A, the left hand side. The vessel was manufactured from PTFE, and it had a total volume of $10 \mathrm{~mL}$. The vessel was filled with $5 \mathrm{~mL}$ cell culture medium, and the droplet generation was performed by pumping PFD into the vessel from the bottom (see red arrow) and contemporary sucking droplets separated by the PFD out of the vessel (see red broken arrow). A specific geometric arrangement of the tubes inside the vessel ensured the reproducible droplet generation. The droplets were guided into PTFE tube with $1.6 \mathrm{~mm}$ inner diameter and the total length of the coil was $3 \mathrm{~m}$ (Fig. 2A, right hand side). The filled coil was sealed with special fittings. The module was mounted on a plate which could be positioned on the shaker if required. The coils of different length could be utilized.

Merging of $800 \mathrm{~nL}$ and $20 \mu \mathrm{L}$ droplets

EBs formation in the $800 \mathrm{~nL}$ droplets was evident after first day of cultivation (Fig. 2B). For prolonged EB cultivation the $800 \mathrm{~nL}$ droplets containing EBs were fused with the $20 \mu \mathrm{L}$ droplets containing medium. 


\section{Cellular Physiology Cell Physiol Biochem 2016;38:1883-1896 \begin{tabular}{ll|l} 
DOI: 10.1159/000445550 & $\begin{array}{l}\text { O 2016 The Author(s). Published by S. Karger AG, Basel } \\
\text { www.karger.com/cpb }\end{array}$
\end{tabular}}

Fig. 2. PBM with modules for $20 \mu \mathrm{L}$ droplet generation and droplet fusion. (A) Module for 20 $\mu \mathrm{L}$ droplet generation (left hand side) and coil for cultivation of cells (right hand side); (B) EB generated within $800 \mathrm{~nL}$ droplet in $1 \mathrm{~mm}$ PTFE tube at day 1 . Scale bar represents $200 \mu \mathrm{m}$; (C) Fusion module for merging of $800 \mathrm{~nL}$ and $20 \mu \mathrm{L}$ droplets.

A module based on a T-junction was developed for droplet fusion (Fig. 2C, left hand side). It is composed of a body made of TECASON (Ensinger GmbH, http://www.ensinger-online. com), PTFE tubes, fittings and 0-rings. TECASON is a transparent polymer which allows controlling the droplet fusion optically. Therefore the live imaging of the droplets fusion procedure could be monitored on PC screen. Pumps for the droplets movements were driven by operator in accordance with the position of the droplets in the tubes.

The $1.0 \mathrm{~mm}$ PTFE tube containing $800 \mathrm{~nL}$ droplets and the $1.6 \mathrm{~mm}$ PTFE tube containing 20 $\mu \mathrm{L}$ droplets were connected to the inputs of the fusion module. Additionally, a $1.6 \mathrm{~mm}$ PTFE tube pre-filled with PFD was connected to the output of the fusion module (Fig. 2C, right hand side).

Droplet fusion was guided by an operator. $20 \mu \mathrm{L}$ droplets were moving continuously through the fusion module by a syringe pump. During the

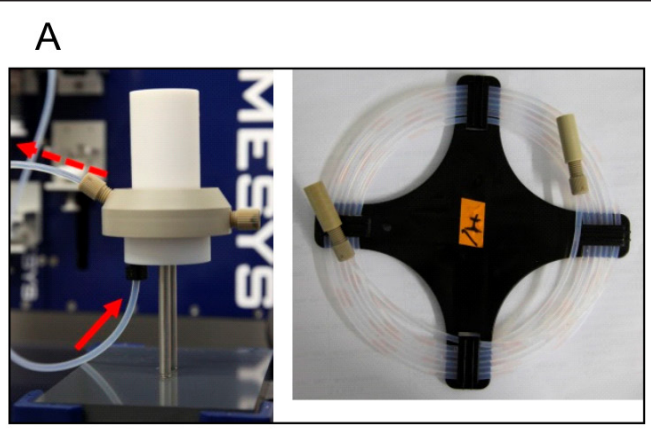

\section{B}

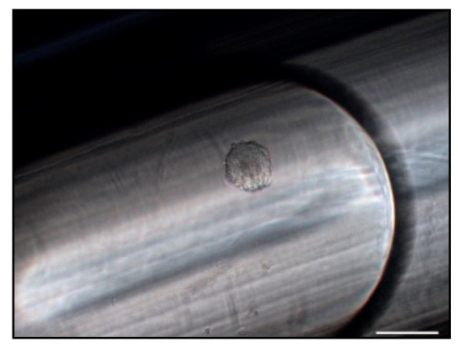

\section{C}

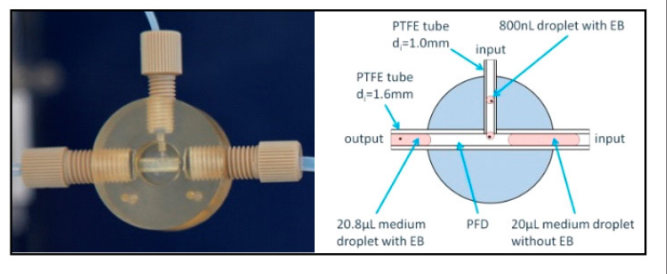

fusion process one $800 \mathrm{~nL}$ droplet was moved by a syringe pump to the direct T-junction immediately after the $20 \mu \mathrm{L}$ droplet had passed the T-junction. The $800 \mathrm{~nL}$ droplet stuck at this T-junction until the fusion with the next $20 \mu \mathrm{L}$ droplet which was coming from the input of the fusion module. Afterword the fused droplet was leaving the T-junction, allowing for the next fusion cycle to occur. The coil containing the fused droplets was incubated in a humidified atmosphere of $5 \% \mathrm{CO}_{2}$ at $37^{\circ} \mathrm{C}$.

\section{Cultivation of ES cells}

Transgenic $\alpha$-PIG44 cell line was derived from mouse D3 ES cells by introduction of both -MHC-EGFP-IRES-PAC and Neo constructs [18]. The former construct is represented by cardiac specific alpha-myosin heavy chain gene promoter, which is directing specific expression of both enhanced green fluorescent protein (EGFP) and puromycin resistance gene. The later construct is directing ubiquitous expression of neomycin resistance gene. The transgenic ES cells were maintained on irradiated Neo resistant mouse embryonic fibroblasts (MEF). The cells were cultivated in Glasgow's Minimum Essential Medium (GMEM) supplemented with $2 \mathrm{mM}$ L-glutamine, 1x non-essential amino acids, $50 \mu \mathrm{M}$ 2-mercaptoethanol, 10\% fetal calf serum (HyClone, http://www.thermoscientific.com), $200 \mu \mathrm{g} / \mathrm{mL}$ G418 (neomycin), and 1000 $\mathrm{U} / \mathrm{ml}$ leukemia inhibitory factor (ESGRO; Millipore, http://www.millipore.com) in a humidified atmosphere of $5 \% \mathrm{CO}_{2}$ at $37^{\circ} \mathrm{C}$. The cells were passaged every second or third day. For the cell passaging the plates with grown ES colonies were washed twice with phosphate-buffered saline (PBS) without $\mathrm{Ca}^{2+}$ and $\mathrm{Mg}^{2+}$ and dissociated with $0.25 \%$ Trypsin/EDTA for $2-3 \mathrm{~min}$ at $37^{\circ} \mathrm{C}$. The enzymatic activity was terminated by addition of double volume of the cell growth medium containing the fetal calf serum. The cells were washed twice with PBS containing $\mathrm{Ca}^{2+}$ and $\mathrm{Mg}^{2+}$ and counted on the electronic cell counter Casy 1 (http:// www.roche.com). All cell culture media and reagents were supplied by Life Technologies (http://www. lifetechnologies.com) unless indicated differently. 


\section{Cellular Physiology Cell Physiol Biochem 2016;38:1883-1896 \\ \begin{tabular}{l|l|l} 
DOI: 10.1159/000445550 & $\begin{array}{l}\text { (C) 2016 The Author(s). Published by S. Karger AG, Basel } \\
\text { www.karger.com/cpb }\end{array}$
\end{tabular}}

Spitkovsky et al.: Generation of Cardiomyocytes in Microbioreactor

\section{Differentiation of ES cells}

ES cell differentiation was performed via EB formation within $1.0 \mathrm{~mm}$ coils. EB formation via standard hanging drop protocol [2] served as a positive reference control. To initiate ES cell differentiation, the cells were harvested as single cell suspension into Iscove's Modified Dulbecco's Media (IMDM) supplemented with 2 mM L-glutamine, 25 mM 4-(2-hydroxyethyl)-1-piperazineethanesulfonic acid (HEPES), 1x non-essential amino acids, $100 \mu \mathrm{M}$ 2-mercaptoethanol and 20\% fetal calf serum. LIF was omitted in the differentiation medium. The cell concentration was either $2.5 \times 10^{4}$ cells/mL for "hanging drop" protocol or $6.25 \times 10^{5}$ cells/mL for PBM. The droplets volumes were either $20 \mu \mathrm{L}$ for "hanging drops" or $800 \mathrm{~nL}$ in PBM so end cell amount under both experimental conditions was 500 cells per compartment. EB formation according to "hanging drop" protocol was initiated on lids of $100 \mathrm{~mm}$ low adhesion dishes. The lids were flipped and placed on the dishes containing $20 \mathrm{~mL}$ PBS to reduce medium evaporation. The hanging drops were cultivated for 1 or 2 days in a humidified atmosphere with $5 \% \mathrm{CO}_{2}$ at $37^{\circ} \mathrm{C}$ followed by suspension culture in the low adhesion plates with 2000 EBs per dish on orbital shaker platform at $20 \mathrm{rpm}$.

\section{Microscopy}

ES cell colony morphology, EBs development, and EBs viability were monitored on Olympus CKX41 inverted microscope under either phase contrast or fluorescent light as appropriate. EBs were collected on $40 \mu \mathrm{m}$ cell strainers, and washed twice with PBS removing both medium and cell debris. EBs viability was determined after stained for $30 \mathrm{~min}$ in dark at ambient temperature in PBS containing $200 \mathrm{nM}$ calcein (Life Technologies, http://www.lifetechnologies.com) detecting live cells and $2.5 \mu \mathrm{M}$ ethidium bromide (SigmaAldrich, http://www.sigmaaldrich.com) detecting dead cells. The microscope stage and camera adjustments were operated via Cell D software (Olympus Soft Imaging Solutions, http://www.olympus-sis.com). The images were acquired with Sony color CCD camera CC-12 (Sony Corporation, http://www.sony.com). At least 10 EBs were captured per experimental point. An average mean diameter of EBs was calculated with measureIT software (Olympus Soft Imaging Solutions, http://www.olympus-sis.com).

\section{Flow Cytometry Analysis}

Either ES cell colonies or differentiated EBs at diverse development stages were dissociated into single cell suspensions with $0.25 \%$ Trypsin/1mM EDTA (Life Technologies, http://www.lifetechnologies. com). Enzymatic treatment time was $5 \mathrm{~min}$ for ES cells or 10-15 min for differentiated EBs, depending on EB size and age. After dissociation, the Trypsin was inactivated with medium containing serum. The cell suspensions were washed twice with PBS and passed through $40 \mu \mathrm{m}$ cell sieves (BD Biosciences, http:// www.bdbiosciences.com) eliminating any cell aggregates. Between $1.0 \times 10^{5}$ and $5 \times 10^{5}$ cells were stained on ice for $30 \mathrm{~min}$. The staining solutions were either PBS with $\mathrm{Ca}^{2+} / \mathrm{Mg}^{2+}$ for cell viability determination or PBS supplemented with $\mathrm{Ca}^{2+} / \mathrm{Mg}^{2+}$ and $0.2 \%$ of human serum albumin (Octapharma, http://www.octapharma. com) for antibody staining. Cell viability was assessed after staining with $1 \mu \mathrm{g} / \mathrm{ml}$ propidium iodide (SigmaAldrich, http://www.sigmaaldrich.com). The antibody staining was conducted either with PE-conjugated anti-SSEA-1 for ES cell "stemness" determination or with PE-conjugated anti-mouse IgM, which was used as the antibody isotype matching control. Both antibodies were from Santa Cruz (http://www.scbt.com). The cells were washed twice in the staining solution after incubation with the antibodies. All stained cell preparations were stored on ice before acquisition. Flow Cytometry of the cells was conducted on FACScan equipped with CellQuest software from BD Biosciences (http://www.bdbisciences.com). The data analysis was performed with Cyflogic software package (http://www.cyflogic.com).

\section{RNA isolation and quantitative reverse transcription polymerase chain reaction}

Total RNA from all samples were extracted with Trizol reagent (Life Technologies, http:// wwwlifetechnologies.com) as recommended by the supplier. RNA from ES cells served as a control. In order to exclude any potential DNA contamination the RNA samples were treated with DNase I (Qiagen, $\mathrm{http}$ // www.qiagen.com) as recommended by the supplier. The isolated RNA concentration and purity was measured on NanoDrop 1000 (Peqlab, http://www.peqlab.com). cDNA were prepared with SuperScript VILO cDNA Synthesis Kit (Life Technologies, http://www.lifetechnologies.com) as recommended by the supplier. In short the reaction was made with $1 \mu \mathrm{g}$ total RNA, $1 \mathrm{x}$ VILO reaction mix and $1 \mathrm{x}$ enzyme mix in 20 $\mu \mathrm{L}$ end volume after incubation at $25^{\circ} \mathrm{C}$ for $10 \mathrm{~min}$, followed by incubation at $42^{\circ} \mathrm{C}$ for $60 \mathrm{~min}$ and the reaction termination at $85^{\circ} \mathrm{C}$ for $5 \mathrm{~min}$. The cDNA was either directly processed for quantitative reverse transcription 


\begin{tabular}{|c|c|c|}
\hline Cellular Physiology & Cell Physiol Biochem 2016; & 8:1883-1896 \\
\hline and Biochemistry & $\begin{array}{l}\text { DOI: } 10.1159 / 000445550 \\
\text { Published online: May 09, } 2016\end{array}$ & $\begin{array}{l}\text { O } 2016 \text { The Author(s). Published by S. Karger AG, Basel } \\
\text { www.karger.com/cpb }\end{array}$ \\
\hline
\end{tabular}

Table 1. Primer sequences used for qRT-PCR analysis

\begin{tabular}{lll}
\hline Gene & Forward Primer & Reverse Primer \\
\hline GAPDH & AGGTCGGTGTGAACGGATTTG & TGTAGACCATGTAGTGAGGTCA \\
NANOG & CCTCAGCCTCCAGCAGATGC & CCGCTTGCACTTCACCCTTTG \\
HAND1 & CAAAAAGACGGATGGTGGTCGC & TGCGCCCTTTAATCCTCTTCTCG \\
Nkx2.5 & CAGCCAAAGACCCTCGGGCG & TGCGCCTGCGAGAAGAGCAC \\
GATA4 & CTCTATCACAAGATGAAGGGCATCAAC & TCTGGCAGTTGGCACAGGAGAG \\
TNNT2 & GGTGCCACCCAAGATCCCCG & AATACGCTGCTGCTCGGCCC \\
\hline
\end{tabular}

polymerase chain reaction (qRT-PCR) or stored at $-80^{\circ} \mathrm{C}$ until needed. qRT-PCR analysis was conducted essentially as described [19]. The real time PCR was performed on ABI 7500 Fast Detection System (Life Technologies, http://www.lifetechnlogies.com) in reaction mixture consisted of $1 \mu \mathrm{L}$ of cDNA, $0.4 \mu \mathrm{M}$ of both direct and reverse primers and MESA FAST qPCR MasterMix Plus for SYBR Assay (Eurogentec, http:// www.eurogentec). The following instrument settings were used: $95^{\circ} \mathrm{C}$ for $5 \mathrm{~min}$ for $\mathrm{cDNA}$ denaturation and Taq hot start polymerase activation followed by 40 cycles of $95^{\circ} \mathrm{C}$ for $15 \mathrm{sec}, 60^{\circ} \mathrm{C}$ for $30 \mathrm{sec}$ and $72^{\circ} \mathrm{C}$ for $30 \mathrm{sec}$. All reactions were performed in triplicates. The primers were designed with Primer 3 software [20]. The following primers were used: GAPDH as a control, Nanog as a stemness maker, Hand1, Gata4, Nkx2.5 and, TNNT2 as cardiac specific markers. The primer sequences are shown in Table 1. The mRNA expression profiles were normalized to GAPDH expression levels with cycle threshold ( $\mathrm{Ct}$ ) method by ABI 7500 Fast Detection System SDS software (Life Technologies, http://www.lifetechnlogies.com).

\section{Results and Discussion}

\section{Generation of EBs within PBM}

Transgenic $\alpha$-PIG44 ES cell line was maintained on neomycin resistant mouse embryonic fibroblasts in the presence of both leukaemia inhibitory factor maintaining the cells in undifferentiated state and G418 to prevent loss of a transgene. The ES colonies had typical sharp borders and were highly condensed as shown on Fig. 3A, left hand side picture. ES cell quality was monitored by Flow Cytometry after staining with antibodies specific for SSEA-1 antigen. As much as $98 \%$ of the cells were SSEA-1 positive as shown on Fig. 3A, right hand side.

We tested a feasibility of EB generation within PBM which was compared with the common hanging drop protocol. According to the hanging drop protocol EB were generated within $20 \mu \mathrm{L}$ droplets loaded with 500 ES cells. However EB formation in $20 \mu \mathrm{L}$ droplets within PBM with $1.6 \mathrm{~mm}$ coils was not optimal due to geometrical constrains sometimes leading to creation of several cell aggregates within a single droplet without a perfect spheroid shape (data not shown), while EB formation was significantly improved within PBM with $1.0 \mathrm{~mm}$ coils with the droplets volumes reduced to $800 \mathrm{~nL}$ while keeping the same initial ES cell number per droplet (500 cells). Under these conditions only single EB were generated and they acquired a smooth spheroid shape already after one day of the cell aggregation initiation (Fig. 2B). Initially the EBs generation and cultivation in the PBM was assessed during 2 days providing direct comparison to EB generation under standard hanging drop protocol. At day $1 \mathrm{EBs}$ formed in $1.0 \mathrm{~mm}$ coils appeared very similar in size and shape to EBs generated under standard hanging drop protocol (Fig. 3B upper panel for the EB morphology and Fig. $3 \mathrm{C}$ for the EB size distribution). The average EB diameter in $1 \mathrm{~mm}$ coils was almost identical to EB in hanging drops as $163.8 \mu \mathrm{m}$ and $163.5 \mu \mathrm{m}$, respectively. During the second day of the cultivation in the PBM, the EB maintained spherical form and continued growing in size, although we noticed some growth retardation as compared to EBs grown in hanging drops (Fig. 3B, lower panel). The average EB diameter was $219 \mu \mathrm{m}$ and $362.8 \mu \mathrm{m}$, respectively. At day 2 the EBs generated under both experimental conditions were released into suspension, and the EB cultivations were continued on low-adhesion plates placed on a shaker. The EBs released from $1 \mathrm{~mm}$ coils resumed growth and interestingly the size difference between the EB generated under 2 different experimental conditions was 
Fig. 3. EB formation within PBM and hanging drops. (A) Morphology of ES cell colonies grown on mouse embryonic fibroblast feeders (left hand side). Scale bar represents $500 \mu \mathrm{m}$. On the right hand side ES cells stained with SSEA-1 antibodies were analyzed by Flow Cytometry, histogram overlay presented SSEA-1 staining vs. staining with isotype matching control antibodies; (B) Morphology and growth of EBs generated under indicated conditions at day 1 (upper panel) and day 2 (lower panel). Scale bar represents $200 \mu \mathrm{m}$; (C) EBs growth kinetic. Average EB diameter is shown, error bars represent standard deviation (SD) and number of samples $(n)=10$. EB size at day 0 was calculated as average volume of a single ES cell at harvest multiplied 500 fold (initial cell load per EB).

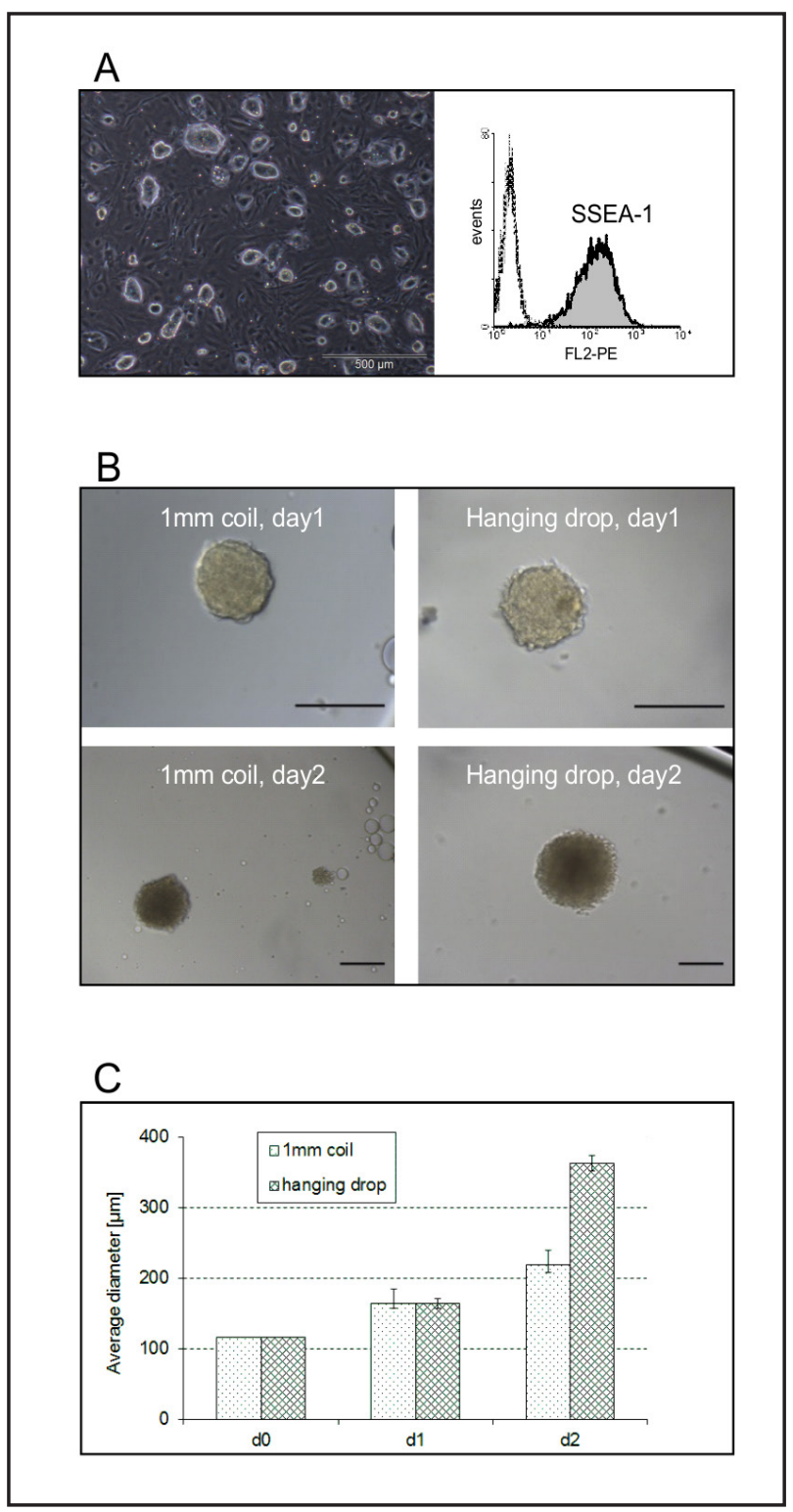

becoming less evident with time (Fig. 4A, upper panel and 4B). At day 5 the average diameter of EBs generated in PBM was $512.1 \mu \mathrm{m}$ compared to $591.5 \mu \mathrm{m}$ for EBs generated in hanging drops. The differences in average diameter by day 8 were further narrowed to $697.4 \mu \mathrm{m}$ and $753.7 \mu \mathrm{m}$, respectively (Fig. 4A, lower panel and 4B). We compared the cell viability within EBs generated under the diverse conditions at day 8. The EBs were enzymatically dispersed into single cell suspensions, and stained with PI dye discriminating live and dead cells. The stained cells were analyzed by Flow Cytometry with results shown in Fig. 4C. EBs generated within PBM contained similar fraction of non-viable cells compared to EBs generated via standard differentiation protocol, or $12 \%$ versus $15.1 \%$ respectively. Most likely non-viable cell fraction reflected ongoing apoptosis coupled to the cell differentiation within EBs under both experimental conditions.

\section{Cardiac differentiation of EBs generated within PBM}

The cardiomyocytes differentiation within EBs could be monitored by the appearance of EGFP which expression was directed by cardiac cell specific alpha myosin heavy chain promoter. Previously we have purified and intensively characterized the $\alpha$-MHC-EGFPpositive cells, and the cell cardiomyocyte fate was proven on a basis of detailed analysis of 

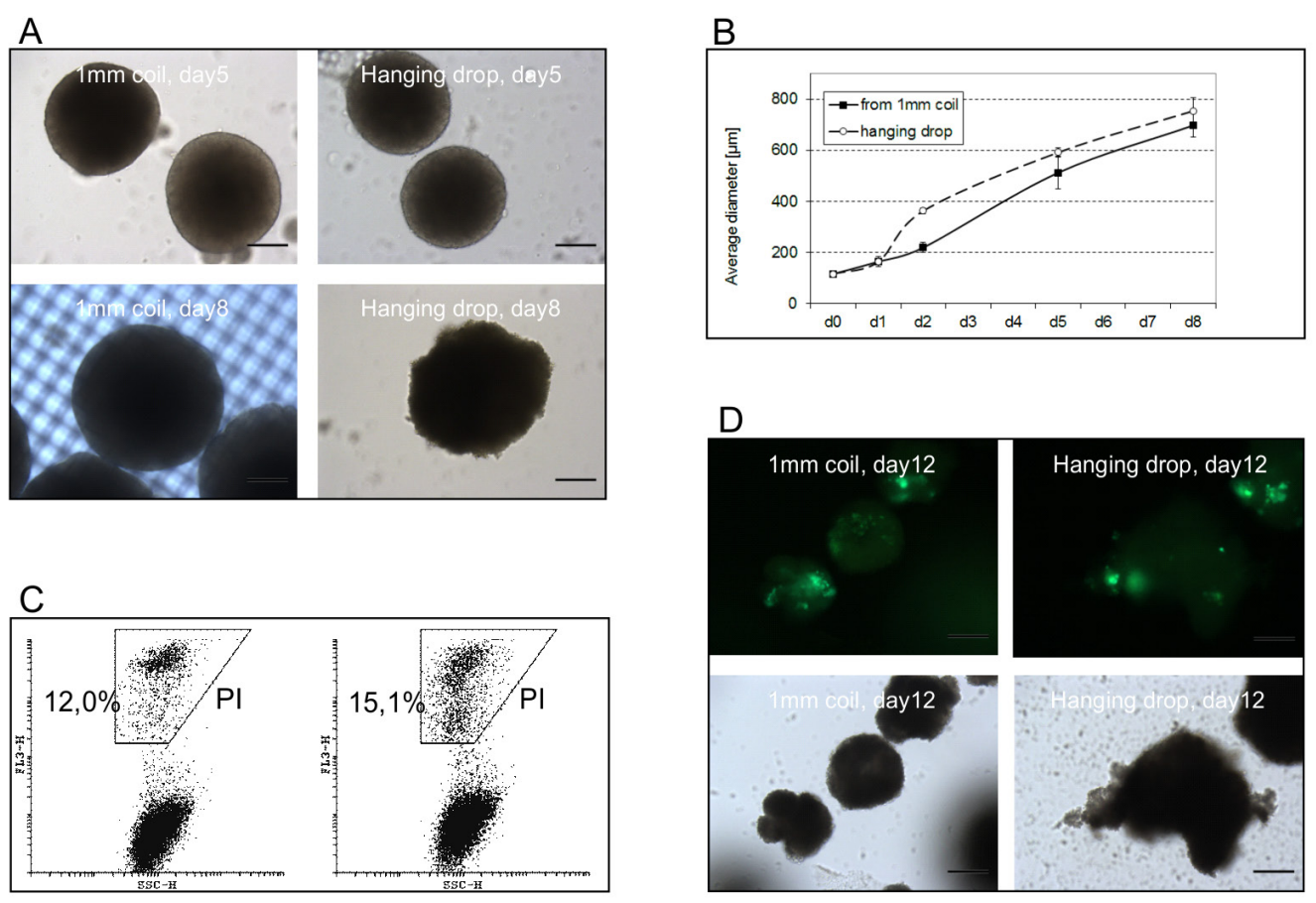

Fig. 4. Cardiac differentiation of EBs generated within PBM. (A) Growth and shape of EBs generated either in PBM (left hand side) or in hanging drops (right hand side) are shown at day 5 (upper panel) and at day 8 (lower panel);(B) Growth kinetic of EBs generated within PBM (left hand side) or in hanging drops (right hand side). Average EB diameter is shown, error bars represent SD and $n=10$. EB size at day 0 was calculated as an average volume of a single ES cell at harvest multiplied 500 fold (initial cell load per EB); (C) Viability of EBs at day 8 generated via alternate protocols was measured by Flow Cytometry non-viable cells shown in gates. EBs generated either in PBM (left hand side) or in hanging drops (right hand side); (D) Clusters of EGFP-positive cardiomyocytes are shown within EBs generated either in PBM (upper left) or in accordance with hanging drop protocol (upper right) could be seen under fluorescent microscope. In the lower panel the bright field microscopy of the corresponding areas is shown. Scale bar represents $200 \mu \mathrm{m}$.

their electrophysiological properties and the specific pattern of the gene expression $[18,21$, 22].

We compared cardiac differentiation of EB generated under either standard hanging drop protocol or within PBM. At day 2 EBs generated under both experimental conditions were released into suspension, and the EB cultivations were continued on low-adhesion plates placed on a shaker as described in a previous section. EGFP-positive areas were emerging already at day 8 in EBs generated under both experimental conditions. The EBs started losing a spherical shape and acquired morphological irregularity after 8 days of cultivation. The EGFP-positive areas within EB continued increasing in size until day 12. Figure 4D, upper panel shows cardiac-specific EGFP-positive areas at day 12 in EBs generated either in PBM (left hand side) or in hanging drops (right hand side). Bright fields of the corresponding EBs are shown on the lower panel. Therefore we could demonstrate that EBs generated in PBM for 2 days were capable in cardiac differentiation after their release into suspension culture.

\section{Continuous EB cultivation and cardiac differentiation within PBM}

Our main aim was to develop a PBM with continuous EB cultivation supporting cardiac differentiation. However $800 \mathrm{~nL}$ droplets used for EBs generation could not provide adequate nutrition for extended period of time. Extended EBs cultivation could be potentially achieved either after medium exchange or after fresh medium re-addition. However the 


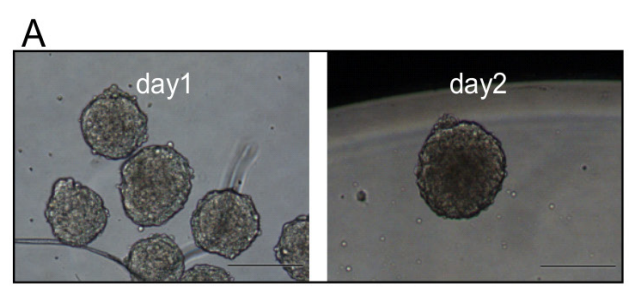

$\mathrm{B}$
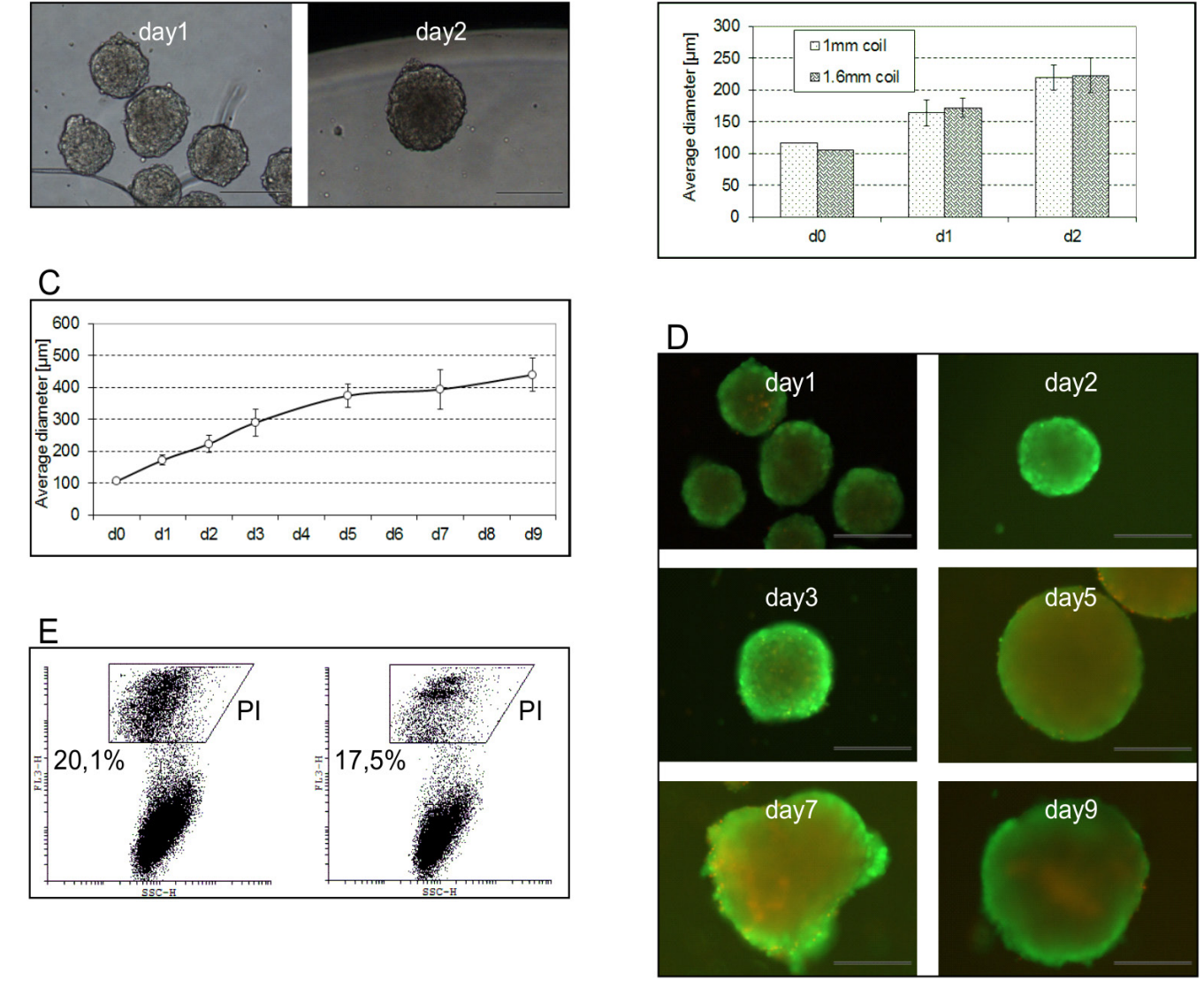

Fig. 5. Continuous EB cultivation within PBM.PBM: (A) EBs at day 1 (left hand side) and at day 2 (right hand side); (B) Comparative growth kinetic of EBs in $800 \mathrm{nl}$ droplets within $1 \mathrm{~mm}$ coil or EBs merged with $20 \mu \mathrm{l}$ droplets within $1.6 \mathrm{~mm}$ coil. Average EB diameter is shown, error bars represent SD and $\mathrm{n}=10$. EB size at day 0 was calculated as an average volume of a single ES cell at harvest multiplied 500 fold (initial cell load per EB); (C) Size distribution of EBs within PBM, average diameter is shown, error bars represent SD and $\mathrm{n}=10$; (D) Live/dead (green/red) staining of EB grown in PBM. Scale bar represents $200 \mu \mathrm{m}$; (E) Viability of EBs at day 8 (left hand side) and day 10 (right hand side) measured by Flow Cytometry. Non-viable cells are shown in gates.

medium exchange for $800 \mathrm{~nL}$ droplets was found to be a complex task while multiple medium exchange cycles were required during EBs cultivation due to small droplet volume constrain. Therefore, we modified the PBM design and EB processing protocol. To this end we introduced an additional step after day 1, which required fusion of $800 \mathrm{~nL}$ droplets with EBs with droplets containing $20 \mu \mathrm{L}$ of cultivation medium. We assumed that such volume of medium could provide adequate EB nutrition until cardiomyocytes differentiation stage. Simple equation is giving support for such assumption. In accordance with the standard EB differentiation protocol up to 2000 EBs originated from hanging drops could be released within $10 \mathrm{~mL}$ cultivation medium and maintained without medium exchange for at least 7-8 days allowing cardiomyocyte differentiation within EBs. It can be calculated as $5 \mu \mathrm{L}$ of medium per EB or at least 4 times lower than droplet volume in the modified PBM.

In summary, the modified EBs processing protocol within PBM consisted of three steps. At the first step, the EBs were formed from 500 ES cells in the $800 \mathrm{~nL}$ droplets within 1 $\mathrm{mm}$ coils and the cultivation was continued for 1 day. Each experimental set up involved at least 3 biological replicates. EBs cultivated for 1 day in in $800 \mathrm{~nL}$ droplets are shown on Fig.

\section{KARGER}


Fig. 6. Continuous growth and cardiac differentiation of EBs in PBM: (A) Fluorescent microscopy of EBs at day 8 (left hand side) with EGFP-positive areas representing cardiomyocytes and the same EBs under bright field microscopy (right hand side). Scale bar represents $200 \mu \mathrm{m}$; (B) Flow Cytometry quantification of cardiomyocytes within EBs at day 8. EGFP-positive cardiomyocytes are shown in the gate; (C) mRNA expression of Nanog, HAND1, Nkx2.5, Gata4 and TNNT2 was defined by qRT-PCR. Relative quantification was performed compared with expression in ES cells in all cases. Error bars represent $\mathrm{SD}$ and $\mathrm{n}=3$.

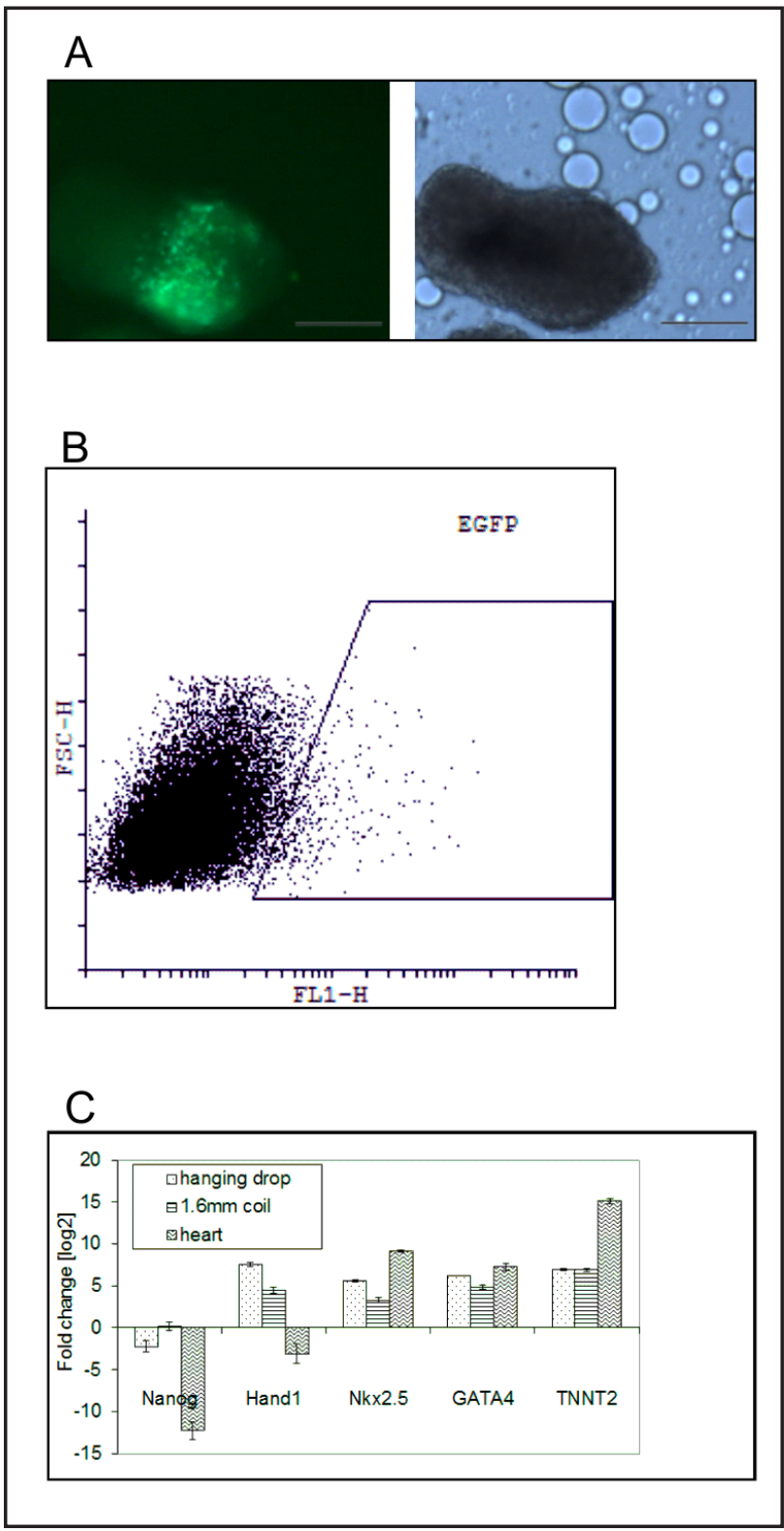

5A, left hand side. At the second step, $20 \mu \mathrm{L}$ medium droplets containing the EB cultivation medium were generated within $1.6 \mathrm{~mm}$ coils. At the third step the droplet fusion procedure was performed after 1 day of EBs incubation in a module merging 800nL droplets with EB with the $20 \mu \mathrm{L}$ medium containing droplets.

The droplets fusion did not affect the EBs appearance, and they continued growing while retaining the spheroid shape EBs released from $800 \mathrm{~nL}$ droplets at day 1 and EB released from a larger $20 \mu \mathrm{L}$ droplet at day 2 are shown on Fig. 5A, left hand side and right hand side, respectively. Interestingly, as shown on Fig. 5B, at day 2 EBs from the fused droplets in the $1.6 \mathrm{~mm}$ coil were similar in size to EBs retained in $1 \mathrm{~mm}$ coil. Therefore, during first 2 days of observation EBs in coils grew slowly compared to EBs in hanging drops independently of the coils diameter (and therefore the droplet volume). This difference in EB growth cannot be explained by nutrition limitation as a fused droplet volume within $1.6 \mathrm{~mm}$ coil was very similar to one within hanging drops. A limited oxygen supply within the coils could potentially explain the difference in EB growth kinetic. Furthermore, surface based forces could be also responsible for modulation of EB proliferation kinetic within coils. Future experiments with additional PBM features allowing oxygen detection within 
droplets could resolve these issues. We continued following the EBs development within $1.6 \mathrm{~mm}$ coils with an aim to achieve cardiomyocyte differentiation stage. In spite of slight growth retardation as compared with a standard hanging drop based protocol, the EBs within PBM continued growing steadily and EB average diameter reached plateau at about $440 \mu \mathrm{m}$ at day 9 (see Fig. 5C). We determined in situ a viability of EB from PMB with live/ dead staining allowing simultaneous detection of viable (green) and non-viable (red) cells. The proportion of the dead cells within EBs became more evident with time; while nonviable cells were almost not-detectable until day 5 (Fig. 5D). Furthermore the cell viability within EBs was also evaluated by Flow Cytometry. The non-viable cell populations consisted of $20.1 \%$ and $17.5 \%$ cells at day 8 and day 10, respectively (Fig. 5E). Slight decrease in number of dead cells detected by day 10 could be explained by reduction of apoptosis rate within developing EBs. The EBs retained spheroid shape in suspension until day 5 (Fig. 5D). The cardiac differentiation potential of differentiating EBs grown in PBM was assessed by appearance of $\alpha$-MHC-EGFP positive cells. EGFP positive areas within EBs could be detected already after day 8 (Fig. 6A, left hand side) or with kinetic similar to one observed in EB processed under standard hanging drop protocol. Cardiomyocytes within developing EBs could be further analysed on a basis of Flow Cytometry of EGFP positive cells detection by Flow Cytometry. Almost 2\% of total cell amount within EBs were EGFP positive by day 8 (Fig. 6B). The proportion of EGFP positive cells was similar to one observed under standard differentiation protocol in these cells at day 8 [18] and data not shown. It is of note that both efficiency of cardiac lineage differentiation and electrophysiological properties of derived cardiomyocytes are cell line dependent and therefore may significantly vary among different ES lines [23]. Cardiac differentiation fate was further confirmed by RT-qPCR analysis of EBs. RNA was isolated from EBs generated either under the standard hanging drop protocol or within PBM at day 8. RNA from mouse ES cells was used as control and mouse heart was used as positive control for cardiomyocytes detection. The particular gene expression levels were determined with the gene specific primer sets (Table 1 and Fig. 6C). Retention of ES "stemness" properties was followed by stem cell specific nanog detection. As expected, nanog was barely detectable in adult mouse heart, and its expression declined in EBs generated via hanging drop protocol. No significant change could be seen in EBs from PBM as compare to non-differentiated ES cells. The results demonstrate that "stemness" phenotype in EBs from PBM decline slowly compared to EBs generated according to hanging drop protocol. Therefore, maturation of EBs in PBM as defined by significant reduction of "stemness" marker expression may require longer cultivation time. In future work we aim to modify cardiomyocyte differentiation protocol for PBM presented here with addition of small molecules and biological factors shown previously to potentiate both efficiency of cardiomyocyte differentiation [24, 25], and their maturation [26].

As to the cardiac cell specific differentiation within EB, cardiac precursor specific marker HAND1 was clearly induced in differentiated EB while it was only barely detectable in adult mouse heart. Two cardiac cell specific transcription factors Nkx2.5 and GATA4 were also clearly induced in EBs; with hanging drop derived EBs expressed them at slightly higher level. Importantly, cardiac Troponin (TNNT2) which is essential for $\mathrm{Ca}^{2+}$ mediated cardiomyocyte contraction was strongly induced and expressed at a similar level between hanging drop derived EBs and EBs generated and maintained within the PBM. The cardiac marker expression within EB generated under our experimental conditions was lower compared to adult heart; however it could be expected since cardiomyocytes within developing EBs represent only a portion of entire cell population.

In summary, the novelty of cardiomyocytes generation presented here has got several distinctive advantages over existing systems for EB manipulation. First of all the EBs remain within closed PBM for entire period of the experiment while in other protocols EB should be transferred between different vessels. Additionally both size and shape of the EBs generated in the PBM are very consistent, a feature, shared with EB generated via standard hanging drop protocol or on plates with surface micropatterning. EBs within PBM withstand even moderate shaking, while EBs within hanging drops or other vessel types remain very fragile 


\section{Cellular Physiology Cell Physiol Biochem 2016;38:1883-1896 \\ \begin{tabular}{l|l} 
DOI: 10.1159/000445550 & $\begin{array}{l}\text { O 2016 The Author(s). Published by S. Karger AG, Basel } \\
\text { www.karger.com/cpb }\end{array}$ \\
\cline { 2 - 3 } & Published online: May 09, 2016
\end{tabular} \\ Spitkovsky et al.: Generation of Cardiomyocytes in Microbioreactor}

and even minimal perturbations may lead to the EBs release or fusion leading to loss of the EB size and shape. Furthermore, the EBs within PBM remain separated into multiple individual compartments for entire period of the EB cultivation making multiple individual assay conditions possible. In relation to 96-well microplates only one $\mathrm{m}$ of $1 \mathrm{~mm}$ coil may hold similar number of individual compartments with $800 \mathrm{~nL}$ droplets, while equal amount of compartments with $20 \mu \mathrm{L}$ droplets may require $3 \mathrm{~m}$ of $1,6 \mathrm{~mm}$ coil. Evaporation in PBM remained neglectable for entire procedure duration providing a tight control of osmolarity in the cultivation medium while evaporation remains a very significant and poorly controlled variable in hanging drop and plate guided protocols. The closed system of EB cultivation has got an advantage of improved safety against undesired contamination and will better meet any regulatory requirements in future. Moreover, the PBM adopts a unique breathable design, providing optimal gas exchange conditions not possible in other devices. Besides PTFE walls permeability for oxygen and carbon dioxide, the droplet separating medium within the coil is highly soluble for oxygen providing additional buffer capacity for steady oxygen supply. PTFE is also reasonably transparent allowing for live monitoring of the internal droplets and their content. Our future work will concentrate on targeted differentiation of human pluripotent cells into cardiac and other specialized cell types, and demonstration of the PBM system applicability for toxicity/drug screening purposes.

\section{Acknowledgements}

This work was supported by the European Commission (HEALTH-2007-1.4-7) under the scope of Hyperlab (High Yield and Performance Stem Cell Lab), Grant agreement no. FP7 - 223011.

\section{Disclosure Statement}

All authors have contributed substantially to this work and declare no conflict of interest.

\section{References}

1 Wu HW, Lin CC, Lee GB: Stem cells in microfluidics. Biomicrofluidics 2011;5:13401.

2 Maltsev VA, Wobus AM, Rohwedel J, Bader M, Hescheler J: Cardiomyocytes differentiated in vitro from embryonic stem cells developmentally express cardiac-specific genes and ionic currents. Circ Res 1994;75:233-244.

3 Schulz JC, Stumpf PS, Katsen-Globa A, Sachinidis A, Hescheler J, Zimmermann H: First steps towards the successful surface-based cultivation of human embryonic stem cells in hanging drop systems. Eng Life Sci 2012;12:584-587.

4 Tung Y-C, Hsiao AY, Allen SG, Torisawa Y-s, Ho M, Takayama S: High-throughput 3D spheroid culture and drug testing using a 384 hanging drop array. Analyst 2011;136:473-478.

5 Ungrin MD, Joshi C, Nica A, Bauwens C, Zandstra PW: Reproducible, Ultra High-Throughput Formation of Multicellular Organization from Single Cell Suspension-Derived Human Embryonic Stem Cell Aggregates. PLoS ONE 2008;3:e1565.

6 Moeller HC, Mian MK, Shrivastava S, Chung BG, Khademhosseini A: A microwell array system for stem cell culture. Biomaterials 2008;29:752-763.

7 Yukawa H, Noguchi H, Hayashi S: Embryonic body formation using the tapered soft stencil for cluster culture device. Biomaterials 2011;32:3729-3738.

8 Torisawa Y-s, Chueh B-h, Huh D, Ramamurthy P, Roth TM, Barald KF, Takayama S: Efficient formation of uniform-sized embryoid bodies using a compartmentalized microchannel device. Lab Chip 2007;7:770776. 


\section{Cellular Physiology Cell Physiol Biochem 2016;38:1883-1896 \begin{tabular}{l|l} 
and Biochemistry Published online: May 09,2016 & $\begin{array}{l}\text { (c) 2016 The Author(s). Published by S. Karger AG, Basel } \\
\text { www.karger.com/cpb }\end{array}$ \\
\hline
\end{tabular} \\ Spitkovsky et al.: Generation of Cardiomyocytes in Microbioreactor}

9 Fung WT, Beyzavi A, Abgrall P, Nguyen NT, Li HY: Microfluidic platform for controlling the differentiation of embryoid bodies. Lab Chip 2009;9:2591-2595.

10 Martin K, Henkel T, Baier V, Grodrian A, Schon T, Roth M, Michael Kohler J, Metze J: Generation of larger numbers of separated microbial populations by cultivation in segmented-flow microdevices. Lab Chip 2003;3:202-207.

11 Grodrian A, Metze J, Henkel T, Martin K, Roth M, Kohler JM: Segmented flow generation by chip reactors for highly parallelized cell cultivation. Biosens Bioelectron 2004;19:1421-1428.

12 Cao J, Kursten D, Schneider S, Knauer A, Gunther PM, Kohler JM: Uncovering toxicological complexity by multi-dimensional screenings in microsegmented flow: modulation of antibiotic interference by nanoparticles. Lab Chip 2012;12:474-484.

13 Funfak A, Brosing A, Brand M, Kohler JM: Micro fluid segment technique for screening and development studies on Danio rerio embryos. Lab Chip 2007;7:1132-1138.

14 Lemke K, Forster T, Romer R, Quade M, Wiedemeier S, Grodrian A, Gastrock G: A modular segmented-flow platform for 3D cell cultivation. J Biotechnol 2015;205:59-69.

15 Witkowski P, Liu Z, Guo Q, Poumian-Ruiz E, Cernea S, Herold K, Hardy MA: Two-layer method in short-term pancreas preservation for successful islet isolation. Transplant Proc 2005;37:3398-3401.

16 Schemberg J, Grodrian A, Romer R, Gastrock G, Lemke K: Online optical detection of food contaminants in microdroplets. Eng Life Sci 2009;9:391-397.

17 Liu WS, Kim HJ, Lucchetta EM, Du WB, Ismagilov RF: Isolation, incubation, and parallel functional testing and identification by FISH of rare microbial single-copy cells from multi-species mixtures using the combination of chemistrode and stochastic confinement. Lab Chip 2009;9:2153-2162.

18 Kolossov E, Lu ZJ, Drobinskaya I, Gassanov N, Duan YQ, Sauer H, Manzke O, Bloch W, Bohlen H, Hescheler J, Fleischmann BK: Identification and characterization of embryonic stem cell-derived pacemaker and atrial cardiomyocytes. Faseb J 2005;19:577-579.

19 Wagh V, Jagtap S, Meganathan K, Potta SP, Winkler J, Hescheler J, Sachinidis A: Effect of chemopreventive agents on differentiation of mouse embryonic stem cells. Front Biosci (Elite Ed) 2012;4:156-168.

20 Rozen S, Skaletsky H: Primer3 on the WWW for general users and for biologist programmers. Methods Mol Biol 2000;132:365-386.

21 Doss MX, Winkler J, Chen S, Hippler-Altenburg R, Sotiriadou I, Halbach M, Pfannkuche K, Liang H, Schulz H, Hummel O, Hubner N, Rottscheidt R, Hescheler J, Sachinidis A: Global transcriptome analysis of murine embryonic stem cell-derived cardiomyocytes. Genome Biol 2007;8:R56.

22 Semmler J, Lehmann M, Pfannkuche K, Reppel M, Hescheler J, Nguemo F: Functional expression and regulation of hyperpolarization-activated cyclic nucleotide-gated channels (HCN) in mouse iPS cell-derived cardiomyocytes after UTF1 -neo selection. Cell Physiol Biochem 2014;34:1199-1215.

23 Hannes T, Wolff M, Doss MX, Pfannkuche K, Haustein M, Muller-Ehmsen J, Sachinidis A, Hescheler J, Khalil M, Halbach M: Electrophysiological characteristics of embryonic stem cell-derived cardiomyocytes are cell line-dependent. Cell Physiol Biochem 2015;35:305-314.

24 Ivanyuk D, Budash G, Zheng Y, Gaspar JA, Chaudhari U, Fatima A, Bahmanpour S, Grin VK, Popandopulo AG, Sachinidis A, Hescheler J, Saric T: Ascorbic Acid-Induced Cardiac Differentiation of Murine Pluripotent Stem Cells: Transcriptional Profiling and Effect of a Small Molecule Synergist of Wnt/beta-Catenin Signaling Pathway. Cell Physiol Biochem 2015;36:810-830.

25 Xu R, Srinivasan SP, Sureshkumar P, Nembo EN, Schafer C, Semmler J, Matzkies M, Albrechtsen M, Hescheler J, Nguemo F: Effects of synthetic neural adhesion molecule mimetic peptides and related proteins on the cardiomyogenic differentiation of mouse embryonic stem cells. Cell Physiol Biochem 2015;35:2437-2450.

26 Wang L, Cui Y, Tang M, Hu X, Luo H, Hescheler J, Xi J: Puerarin facilitates T-tubule development of murine embryonic stem cell-derived cardiomyocytes. Cell Physiol Biochem 2014;34:383-392. 